\title{
Impacto psicosocial del diagnóstico del Trastorno del Espectro Autista (TEA) en los principales cuidadores.
}

\author{
Paulina Abadia Alvarado ${ }^{1} \&$ Virginia Torres-Lista ${ }^{1,2,3 *}$. \\ ${ }^{1}$ Universidad Católica Santa María La Antigua (USMA), ${ }^{2}$ Sistema Nacional de Investigación (SNI), \\ ${ }^{3}$ Centro de Investigaciones Científicas de Ciencias Sociales (CENICS).
}

*Autor para correspondencia. Email: vtorresl@usma.com.pa

Recibido: 12 de marzo de 2019

Aceptado: 11 de abril de 2019

\begin{abstract}
Resumen
El Trastorno del Espectro Autista (TEA), está clasificado como Trastorno del desarrollo Neurológico, esta condición se caracteriza por una perturbación grave y generalizada en diversas áreas del desarrollo como: las habilidades sociales, la comunicación e intereses o conductas estereotipadas. La sospecha del Autismo se puede iniciar antes del primero año de vida, sin embargo, el diagnóstico en algunos casos es tardío trayendo como consecuencia un impacto significativo en diferentes áreas de la vida personal, familiar, de pareja, social, psicológica y económica de todo el grupo familiar. Esta repercusión modifica el estilo de vida generando procesos de duelo que pueden tener un desarrollo negativo o positivo de acuerdo a la intervención temprana tanto para el niño como a los familiares. Es por esto que esta investigación busca comprender el impacto psicosocial sobre el diagnóstico del Trastorno del Espectro Autista (TEA) en los padres o cuidadores, mediante estudios de casos realizados en El Centro Ann Sullivan Panamá. Este trabajo tiene un diseño mixto con mayor énfasis cualitativo, donde cuenta con estudios de casos múltiples, con el propósito de entender el impacto que ha causado en los padres o cuidadores tener un hijo (a) con Autismo. En esta investigación se encontró que hubo un impacto elevado, en el momento del diagnóstico, es decir, presentaron etapas de vulnerabilidad, en tres de las cuatro áreas (socioeconómico, sociopsicológico, funcionamiento familiar). Donde las áreas más sensibles fueron la sociopsicológica (integración social, participación social, modo de vida familiar, convivencia social, planes y proyectos filiares) y las funciones familiares (comunicación familiar, afectividad, cohesión, armonía, roles). Por lo tanto, la sociedad panameña debe seguir informándose acerca del Autismo, para permitir mayor concientización, así como también mejorar la integración social tanto del niño como la familia en general, poder disminuir las creencias/mitos erróneos con respecto al TEA.
\end{abstract}

Palabras Clave: Autismo, Familia, Diagnóstico, Estudio de casos, Psicosocial.

\section{Abstract}

The Autism Spectrum Disorder (ASD), is classified as Neurological Development Disorder, this condition is characterized by a serious and widespread disturbance in the areas of development such as: social skills, communication and stereotypical interests or behaviors. The suspicion of autism can 
Invest. pens. crit. (ISSN 1812-3864)

Vol. 7, No. 1, enero- abril 2019

pp. 27-39

be initiated before the first year of life, however, the diagnosis in some cases, time, attention, impact, impact in the areas of personal, family, social, psychological and economic life of everything. The family group This repercussion modifies the way of life generating bereavement processes that can have a negative or positive development according to the early intervention as well as the child and the relatives. This is why this research seeks to understand the psychosocial impact on the diagnosis of Autistic Spectrum Disorder (ASD) in parents and caregivers, through case studies conducted at The Ann Sullivan Panama Center. This work has a design that combines with greater qualitative emphasis, where it has multiple case studies, in order to understand the impact that has occurred in parents or caregivers having a child with Autism. In this research it was found that there was a high impact, at the time of diagnosis, that is, the stages of vulnerability, in three of the areas (socioeconomic, socio-psychological, family functioning). Where the most sensitive areas were the socio-psychological (social integration, social participation, family lifestyle, social coexistence, plans and filial projects) and family functions (family communication, affectivity, cohesion, harmony, roles). Therefore, Panamanian society should continue to be informed about Autism, to allow greater awareness, besides improve the social integration of both the child and the family in general, to reduce the erroneous beliefs/myths regarding ASD.

Key words: autism, family, diagnosis, case study, psychosocial.

\section{Introducción}

El Trastorno del Espectro Autista (TEA), se encuentra clasificado bajo el título Trastorno del desarrollo Neurológico. Esta condición se caracteriza por una perturbación grave y generalizada en diversas áreas del desarrollo como son: las habilidades sociales, habilidades para la comunicación, intereses o conductas estereotipadas. Estas características por lo general se presentan durante los primeros años de vida, pero se tienden a confundir con una discapacidad intelectual, alguna enfermedad médica u otras afectaciones. (APA, 2014; Martínez, A., \& Borges, A., 2012).

Asimismo, el TEA es diagnosticado usualmente después de los tres (3) años de edad. Una de las razones es que se cree que las características o las sintomatologías no se llegan a observar/percibir sino hasta un tiempo después del nacimiento. (Canal et al., 2006). Sin embargo, se puede detectar a tempranas edades: la ausencia de gestos comunicativos, no mirar hacia donde otros señalan, alteraciones en la interacción social, en las respuestas emocionales y en el juego, escaso interés en las personas, intereses restringidos, movimientos o habla repetitivos (estereotipados), híper o hipo reactividad a los estímulos sensoriales o intereses inhabituales por aspectos sensoriales del ambiente, falta de flexibilidad en rutinas, entre otros (APA, 2014; Taylor, Smiley y Richards, 2009).

No obstante, Canal et al., (2006) señala que, a pesar de existir similitudes en cuanto a las deficiencias de las interacciones sociales, no existe una única conducta que siempre sea típica del Autismo o de cualquiera de los Trastornos del Espectro Autista. La presencia de estas características debe tomarse como un llamado de atención, es decir, no deben pasarse por alto, como si las mismas fueran parte del proceso de desarrollo. Al notar estos elementos de manera temprana, puede facilitar el proceso de diagnóstico e intervención.

\subsection{Creencias erróneas sobre el Trastorno del Espectro Autista (TEA)}

Las creencias son modelos cognitivos utilizados por las personas para adaptarse al medio, e impactan en la manera de sentir y actuar. Se consideran erróneas cuando no son apoyadas por los 
Invest. pens. crit. (ISSN 1812-3864)

Vol. 7, No. 1, enero- abril 2019

pp. 27-39

resultados científicos de la época. (Valdés Cuervo, et al. 2009) Actualmente, muchos individuos presentan creencias erróneas sobre el TEA, esto se debe a la falta de información y el escaso conocimiento que se tiene del mismo. Al contar con información falsa, esta dificulta los diagnósticos, la atención y los cuidados de los niños. Valdés Cuervo, et al. (2009) y Álvarez-López et al. (2014) identificaron las principales creencias erróneas por parte de los padres con hijos con Trastorno del Espectro Autista, como son la etiología, características y tratamiento:

\section{a) Creencias sobre la etiología:}

- Factores hereditarios: El TEA no tiene una etiología concreta; sin embargo, diversos estudios han proporcionado información respecto a algunas anomalías genéticas que comparten individuos con TEA. Solo el 10\% de los casos con Autismo se deben a alteraciones de un único gen 1p21.3 y anomalías citogenéticas. El descubrimiento de mutaciones en los genes que codifican las proteínas postsinápticas neuroliginas (NL4 y NL3) y de anclaje (SHANK2 y SHANK3), podrían participar en la neurobiología del Autismo.

- Problemas en el parto: A su vez se vincula a ciertas condiciones al parto como sufrimiento fetal que puede estar asociado a lesiones que podrían afectar el nivel cognitivo del niño, pero, este sufrimiento no es una causa que se relacione estrictamente con esta condición.

- Vacunas: La vacuna Triple Viral han sido otra creencia errónea que por mucho tiempo se le ha prestado atención. Debido al polémico estudio publicado en la revista 'The Lancet' en 1998 que fue retractado una década después de la publicación. Un nuevo estudio realizado con más de 650.000 niños daneses nacidos entre 1999 y 2010 y seguidos durante más de una década. Encontraron, que los niños que reciben la vacuna contra el sarampión, las paperas y la rubéola (MMR, por sus siglas en inglés) no tienen mayor riesgo de autismo, y eso incluye a los niños que se considera que están en grupos de "alto riesgo" para el trastorno del desarrollo neurológico. (Hviid A, et al., 2019).

- Trato y edad avanzada de los padres: Otra de las más escuchadas creencias es el trato frio de los padres, pensando que si la madre es distante y poco afectiva con su hijo(a), este puede desarrollar Autismo. Como mito, se puede mencionar adicionalmente la edad avanzada de los padres y el estrés durante el embarazo como posibles causantes.

\section{b) Creencias sobre las características:}

- Son niños superdotados: Se cree que los individuos con TEA siempre tienen una inteligencia promedio o superior del promedio. Esta información circula, debido a los estereotipos adquiridos mediante películas que muestran a personas con Autismo vinculados como niños genios "sindrome del sabio", esto ocurre 1 de cada 10 niños con Autismo. Cada niño con esta condición es único y la inteligencia varia ampliamente de uno a otro por lo que es necesario reconocer las diferencias individuales.

- Adquisición del lenguaje: Se cree que los niños a los dos años de edad no poseen un lenguaje comunicativo tienen Autismo. A pesar de que esto sea una característica del trastorno, no se puede atribuir este rasgo únicamente al hecho de que el individuo tenga TEA. Se deben tomar en cuenta otras características, debido que algunos niños hablan tarde o puede ser señal de otra afectación que no necesariamente sea esta. Pero, es importante indicar que el $75 \%$ de los niños con Autismo presentan retraso mental.

- Berrinches y agresiones debido a la malacrianza: Muchas veces los cuidadores olvidan que esto se da debido a los problemas en su funcionamiento ejecutivo, es decir; es una manifestación 
Invest. pens. crit. (ISSN 1812-3864)

Vol. 7, No. 1, enero- abril 2019

pp. 27-39

de su dificultad para planificar, organizar, cambiar en forma repentina a nuevos esquemas mentales que alteren sus rutinas, y de la dificultad de aislarse de los estímulos que les provocan dichas reacciones.

- En cuanto a la dependencia de los padres por toda la vida: Se puede decir que eso depende del cada caso, ya que existen individuos que han podido llevar una vida de autosuficiencia.

\section{c) Creencias sobre el tratamiento:}

- El Autismo se cura con tratamiento y medicamentos: El Autismo es un síndrome clínico y no una enfermedad curable. Los síntomas en el/la niña(a) diagnosticado pueden tratarse y disminuirse con medicamentos neurolépticos, antidepresivos, y benzodiacepinas, los cuales ayudan a disminuir la sintomatología típica del autista. Se piensa que el tratamiento es capaz de curar esta condición, al mismo tiempo se le da valor a la medicina alternativa, como también a la terapia con animales para producir mejoramientos. Los medicamentos también pueden y deben combinarse tanto con terapia psicológica como psiquiátrica para ofrecerle una mejor calidad de vida tanto al paciente como a sus familiares y cuidadores.

Debido que el TEA es un síndrome multifactorial, generando una incidencia de mitos y creencias respecto a su etiología, sintomatología, diagnóstico y pronóstico que dan pie a numerosas suposiciones, las cuales pueden ser erradas (Álvarez-López, et al., 2014). Por otro lado, es importante que los padres cuenten con una red de apoyo adecuada porque muchas veces los medios de comunicación e internet (la más consultada por parte de los padres), permiten la difusión de información la cual puede ser empírica o simplemente mitos, que pueden obstaculizar la intervención temprana de los niños.

\subsection{Factores psicosociales que intervienen en el diagnóstico del TEA}

El diagnóstico del TEA, no solo afecta al individuo, sino también a la dinámica familiar. Además, el diagnóstico conlleva cambios significativos en los miembros que integran la familia, su modo de funcionar o vivir (tanto económico como social), puede aparecer repentinamente, generando modificaciones de las reglas y roles, se reviven conflictos no resueltos, aumenta la tensión entre los miembros de la familia, problemas en la convivencia (matrimonial/familiar), conductas no solidarias por parte de algún miembro en la familia, ausencia de intimidad, falta de espacio, desgaste emocional, gastos económicos excesivos, auto-atribución y atribución de las tareas y roles entre el padre y la madre, el cuidado del niño, grado de sensibilidad hacia los problemas que presenta el niño, la percepción de los padres frente al trastorno y el entorno social, entre otros. (Martínez \& Borges, 2012) Estos eventos pueden estar acompañados por sentimientos de frustración, estrés, ansiedad, entre otros.

Por otro lado, otros integrantes de la familia se pueden ver afectados, en este caso los hermanos, debido que se pueden presentar sentimientos ambivalentes hacia sus padres si estos dedican más tiempo a su hermano/a y creen que han sido abandonados. También pueden presentar autoestima baja, temprana independencia, vergüenza hacia su hermano(a) y restricción en las relaciones interpersonales (Ávila, F., et al., 2006), incluso pueden llegar a pensar que sus padres son sobreprotectores con su hermano(a) con TEA. Sin embargo, dependiendo de la edad del hermano no siempre el/la niño(a) puede comprender la complejidad del tema sino hasta que tenga una edad mayor. 
Invest. pens. crit. (ISSN 1812-3864)

Vol. 7, No. 1, enero- abril 2019

pp. $27-39$

En cuanto al impacto sociofamiliar, Mesa-Hernandez, F. (2015) menciona que a nivel laboral uno de los cuidadores principales, tiene que reducir las jornadas de trabajo y muchas veces dejarla definitivamente. Esto ocurre por la necesidad de cumplir con las responsabilidades diarias de su hijo(a). Es importante mencionar, no todas las situaciones son iguales, se conocen casos en los cuales el desempeño laboral, ya sea de la madre o del padre tiende a descender debido al fuerte cansancio tanto físico y emocional que conlleva el cuidado, generando muchas veces el síndrome del cuidador.

Por otra parte, a nivel económico también se evidencian repercusiones debido al esfuerzo financiero, producido por los gastos de los viajes (traslado de otras ciudades), acudir a diversas consultas (especialistas), tratamiento farmacológico y terapia de estimulación. Otros factores repercutidos son las relaciones sociales (amistades/otros miembros familiares), debido que hay una reducción de las mismas, muchas veces esto ocurre por el proceso de exclusión de involucrar a padres con hijos con Autismo a la hora de realizar planes recreativos, pues en ocasiones manifiestan que los niños son inquietos y los demás pueden percibirlo de una manera incómoda o molesta.

Sin embargo, cada familia es única, dicha experiencia puede ser vivida en diferentes formas, mientras una familia se puede adaptar en corto plazo otra puede pasar por un proceso más prolongado. Así mismo, uno de los miembros que se ve mayormente afectado es la madre, debido que muchas veces es en ella en quien recae la responsabilidad del hijo(a).

\subsection{Situación Actual del TEA en Latinoamérica}

En la actualidad se calcula que 1 de cada 160 niños se ven afectados con el Trastorno del Espectro Autista (TEA), sin embargo, esta cifra puede variar debido al ingreso económico y el nivel sociocultural de cada país (OMS, 2016). En Latinoamérica no se cuentan con estudios de prevalencia del TEA, pero la cifra próxima es de 1 de cada 100 personas. (Stamboulian, 2015). Esta ambivalencia en la estadística es debido que los estudios son escasos, los artículos publicados en estas regiones son principalmente de corte teórico y la mayoría de datos epidemiológicos que se manejan provienen de población inglesa y estadounidense (Verán C, N. 2011; Mebarak, M., Martínez, M. \& Cruz, M. (2008).

No obstante, los datos epidemiológicos que se manejan son variados, por ejemplo, Levy et al. (2009) plantean que la prevalencia es entre 25 y 116 por cada 10.000 niños, mientras Baron-Cohen (2008) manifiesta que un 1\% de la población posee rasgos propios del Trastornos. En Brasil, en 1997 se estimó que había aproximadamente 600 mil personas de la población total (Bossa \& Callias, 2000); en el 2008, en Venezuela, la prevalencia del TEA era de 17 por cada 10.000 niños entre 3 y 9 años de edad (Montiel-Nava \& Peña, 2008)

Por otra parte, en Panamá para el 2013 se contabilizó que había 686 casos de niños(as) con TEA (Ministerio de Salud, 2013; Lasso, M., 2013), sin embargo, entre el 2014 y 2016, en Panamá, de acuerdo con la información recopilada por el Sistema de Estadísticas de Salud (SIES) se atendieron 629 personas con autismo con un rango de edad de 1 a 4 años, 5 a 9 años y de 10 a 14 años. Además, hay una prevalencia de acuerdo al género, es cuatro veces mayor en hombres que en mujeres (López et al., 2009). 
Invest. pens. crit. (ISSN 1812-3864)

Vol. 7, No. 1, enero- abril 2019

pp. 27-39

\section{Materiales y Métodos}

La presente investigación es un estudio mixto, con mayor énfasis cualitativo, donde se analizó estudios de casos múltiples a través de aspectos cuantitativos/cualitativos. Respecto a su propósito, las investigaciones realizadas a través del método de estudio de caso pueden ser: descriptivas, si se pretende identificar y describir los distintos factores que ejercen influencia en el fenómeno estudiado (El Autismo), y explicativo, porque busca explorar variables adyacentes que hayan influido en el diagnóstico. (Hernández, et al, 2010). Además, por su carácter mixto, se recogió la información desde distintas fuentes tanto cualitativas como cuantitativas, como: documentos, registros de archivos, entrevistas directas, observación directa, cuestionarios, observación de los participantes e instalaciones u objetos físicos. (Yin, 1989; Martínez Carazo, P.C, 2006; Hernández, et al, 2010).

Como instrumento de valoración se utilizó el Cuestionario para Familias de Personas con Autismo, compuesto por 43 preguntas, en él se recogen datos sobre las necesidades de las personas con autismo y sus familias en cuanto al diagnóstico, atención y orientación (Instituto de Salud Carlos III, 2015). Además, se utilizó El Instrumento de Repercusión Familiar (IRFA), es un instrumento de 20 ítems, distribuidos por áreas que evalúan variables específicas como: Socioeconómico, Sociopsicológico, Funcionamiento familiar, Estado de salud. Según la respuesta del consenso familiar se circuló el número de acuerdo al grado en que el evento produjo cambios en la familia y se señaló en forma positiva o negativa; favorable $(+)$ o desfavorable (-). Este instrumento presenta una validez de contenido, criterio y constructo, que determina la confiabilidad del mismo. (Herrera, P., 2002).

La muestra estuvo conformada por ocho (8) cuidadores principales (hombres y mujeres) de figuras parentales (padre-madre; madre; abuelo) de niños y niñas diagnosticados con TEA que asisten al Centro Ann Sullivan de la Ciudad de Panamá. El rango de edad de los niños con TEA es de (15.2 años \pm 0.85). Por otro lado, la información obtenida, fue procesada con el software SPSS $®$ versión 24. Debido a su característica de la distribución de los datos, estos se analizaron de manera no paramétrica, utilizando la mediana como estadístico descriptivo para el análisis de las áreas. Además, para determinar la asociación entre las áreas de repercusión del IRFA, se hizo un análisis con la prueba de Friedman y una correlacional de Rho de Spearman, donde en todas las pruebas, $P<0.05$ fue considerado estadísticamente significativo.

Consideraciones Éticas: Para este estudio se consideraron las pautas éticas establecidas por la Organización de las Naciones Unidas para la Educación, la Ciencia y la Cultura, bajo el título Declaración Universal sobre Bioética y Derechos Humanos (2005). Asimismo, los participantes fueron informados acerca de la confidencialidad del proceso de investigación (respeto a la privacidad/confidencialidad). Adicionalmente, la presente investigación cumple con los criterios que propone Las Buenas Prácticas en Investigación presentadas por la Universidad Católica Santa María la Antigua. Se ha respetado el principio de autonomía, bienestar, no malevolencia, justicia, integridad y responsabilidad social y ambiental (Universidad Católica Santa María La Antigua, 2012).

\section{Resultados}

Los resultados encontrados en el Instrumento de Repercusión Familiar (IRFA), indican que hubo una repercusión Elevada con una Mediana $(\mathrm{Me}=76.5)$ (Gráfica 1) colocando de manifiesto a los individuos (núcleo familiar) durante el momento del diagnóstico los cuales estuvieron expuestos a un contexto 
Invest. pens. crit. (ISSN 1812-3864)

Vol. 7, No. 1, enero- abril 2019

pp. 27-39

de vulnerabilidad. Con la finalidad de explorar la asociación entre las áreas del test, se analizó la Prueba Friedman, donde se encontró que las áreas de (repercusión socioeconómica, repercusión sociopsicológica, funcionamiento familiar y estado de salud), están asociadas de forma significativa $\left(\chi 2_{(4)}=30.886, \mathrm{P}<0.001\right)$.

Por otra parte, donde el área con mayor impacto fue el sociopsicológica y funciones familiares, con un nivel (Severo). No obstante, el nivel socioeconómico y el estado de salud, fue un nivel entre (Moderado a Elevado). Estos resultados indican que, el núcleo familiar ante el diagnóstico del TEA, vivieron momentos de estrés, ansiedad, etc., que ejercieron un cambio en la dinámica/rol familiar, estilos de vida, reajuste de las finanzas. No obstante, la evaluación del sentido favorable o desfavorable, se situó ( $62 \%$ positivo vs. 38\% negativo), mostrando, que ante los cambios que ejerce el TEA, las familias pudieron ajustarse de forma positiva (mediano a largo plazo) a los cambios, es decir, buscaron orientación profesional, ayuda, entre otros elementos, que contribuyen a la adaptabilidad social de todos los individuos.

$\mathrm{Al}$ analizar el coeficiente de correlación entre los factores, se observó una relación positiva entre el Total Global del IRFA con tres de las áreas de repercusión, (área socioeconómica, Rho de Spearman, $\mathrm{r}=0.713, \mathrm{P}<0.01$; área sociopsicológica, Rho de Spearman, $\mathrm{r}=0.928, \mathrm{P}<0.01$; y funcionamiento familiar, Rho de Spearman, $\mathrm{r}=0.963, \mathrm{P}<0.01)$. Por otro lado, el área sociopsicológica esta correlacionada con el funcionamiento familiar (Rho de Spearman, $\mathrm{r}=0.889, \mathrm{P}<0.01$ ) (Gráfica 1).

Gráfica 1. Repercusión Familiar ante el diagnóstico de TEA.

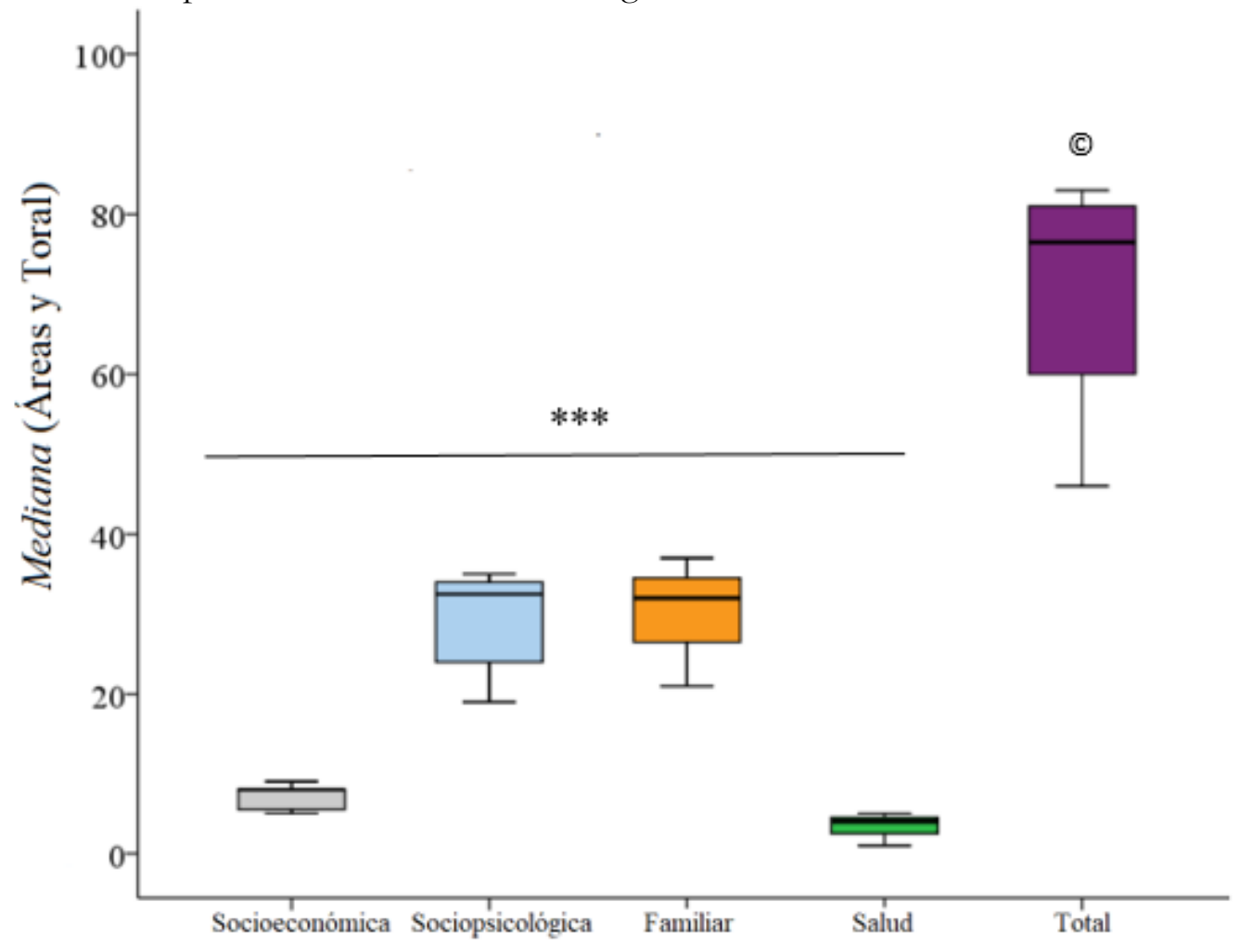


Invest. pens. crit. (ISSN 1812-3864)

Vol. 7, No. 1, enero- abril 2019

pp. $27-39$

Gráfica 1. Repercusión Familiar ante el diagnóstico de TEA. Los datos están expresados en mediana, para evaluar las cuatro (4) áreas de repercusión: Área socioeconómica $(\mathrm{Me}=8)$, Área Sociopsicológica $(\mathrm{Me}=32.50)$, Funcionamiento familiar $(\mathrm{Me}=32.00)$ y Estado de salud $(\mathrm{Me}=4)$. Adicional, se incorpora el Total Global $(\mathrm{Me}=76.50)$ obtenido en el I (C) . Prueba Friedman, $* * * \mathrm{P}<0.001$. Correlación positiva $(\mathrm{C}$ de Spearman del Total global del IRFA con el área socioeconómica, sociopsicológica y función familiar, $\mathrm{P}<0.01$ )

Por otro lado, de acuerdo al cuestionario para familias de personas con autismo, se observó que el cuidador principal es la madre (50\%), el 220/ madre-padre y el 17\% abuelo. Donde el 83\% de los casos eran niños con respecto al 17\% que er $* * *$; con TEA. El 34\% de los niños/as se encuentran en la etapa de la infancia con respecto al $33 \%$ que se hallan entre la adolescencia y la adultez joven. La media y desviación típica de edad de los niños/as es de (15.2 años \pm 0.85$)$.

En la Tabla 1, se reflejan los resultados de las variables sociodemográfica y del diagnóstico del TEA, donde se observa que el 100\% de los casos viven en un entorno urbano, en vivienda unifamiliar. El $67 \%$ de las familias tienes una estructura monoparental, con respecto al 33\% en estructura nuclear.

Tabla 1. Variables sociodemográficas y del diagnóstico del TEA.

\begin{tabular}{|c|c|}
\hline Variables & $\begin{array}{l}\text { Porcentaje } \\
(\%)\end{array}$ \\
\hline \multicolumn{2}{|l|}{ Variables Sociodemográficas } \\
\hline \multicolumn{2}{|l|}{ Tipo de vivienda (residencia actual) } \\
\hline Apartamento & $33 \%$ \\
\hline Casa & $50 \%$ \\
\hline \multicolumn{2}{|l|}{ Unidad Familiar } \\
\hline 2 a 4 personas & $34 \%$ \\
\hline 5 a 6 personas & $67 \%$ \\
\hline \multicolumn{2}{|l|}{ Ingreso Anual } \\
\hline$\$ 2000$ a $\$ 8000$ & $33 \%$ \\
\hline$>\$ 12000$ & $17 \%$ \\
\hline \multicolumn{2}{|c|}{ Variables asociadas al diagnóstico del TEA } \\
\hline \multicolumn{2}{|c|}{ Edad del niño al momento de las primeras sospechas de TE $A$} \\
\hline$<1$ año de edad & $17 \%$ \\
\hline$>1$ a 3 años de edad & $83 \%$ \\
\hline \multicolumn{2}{|l|}{ Primera persona en sospechar } \\
\hline Madre & $50 \%$ \\
\hline Otro Familiar (Abuela, tíos, etc.) & $33 \%$ \\
\hline Pediatra & $17 \%$ \\
\hline \multicolumn{2}{|l|}{ Primera consulta con el diagnóstico (Edad) } \\
\hline$>1$ año y 6 meses & $50 \%$ \\
\hline$>2$ a 4 años de edad & $50 \%$ \\
\hline \multicolumn{2}{|l|}{ Tipo de atención (Consulta) } \\
\hline Atención primaria (Pediatría) & $60 \%$ \\
\hline Neurología & $30 \%$ \\
\hline
\end{tabular}


Invest. pens. crit. (ISSN 1812-3864)

Vol. 7, No. 1, enero- abril 2019

pp. $27-39$

\begin{tabular}{lc}
\hline \hline Atención psicológica & $10 \%$ \\
Antecedente de TEA en la familia & \\
Sí & $67 \%$ \\
No & $33 \%$ \\
Aspectos importantes durante el diagnóstico (para la familia) & \\
Comunicación del diagnóstico & $90 \%$ \\
Trato recibido (atención) & $88 \%$ \\
Asesorías (información) & $87 \%$ \\
Tiempo de espera & $78 \%$ \\
\hline
\end{tabular}

La variable asociada al diagnóstico es básica en este estudio de casos, ésta define la situación de impacto a la que debe adaptarse el sujeto y familia. Donde se encontró lo siguiente: respecto a las primeras sospechas del TEA o que algo ocurría con los niños/as se encontró que el 83\% de los casos tuvieron sus primeras preocupaciones después del primer año de vida, con respecto al 17\% que ocurrió antes del primer año de vida del niño. La primera persona en darse cuenta del problema en el $50 \%$ fue la madre.

"No hablaba, se mecía, se mordia los dedos..." se golpeaba la cabeza, conducta de hiperactividad, poco afectivo, movimientos estereotipados".

No obstante, la primera consulta al profesional de la salud fue al año y seis meses representado por el $50 \%$ mientras que el otro 50\% de los casos acudieron al especialista entre los 2 a 4 años de edad. Además, el 100\% de los casos manifestó que el especialista que dio con el diagnóstico definitivo fue el Pediatra, Neurólogo y Neuropsiquiatra, sin embargo, la tardanza entre el tiempo de la primera sospecha al diagnóstico definitivo, en algunos casos fue por los múltiples diagnósticos previos como: hiperactividad, problemas de memoria a corto plazo, dislexia y retraso mental. Asimismo, los niños/as con TEA se les realizó múltiples pruebas antes, durante y después del diagnóstico (pruebas de audición, psicológicas y metabólicas).

Además, la asistencia sanitaria fue $50 \%$ pública y $50 \%$ privada, donde los padres o acudientes indicaron que las consultas privadas eran porque se encontraban satisfechos con la información, red de apoyo y atención, sin embargo, los niveles de satisfacción (13\%) disminuían en el sistema público, debido al tiempo de espera para ser atendido (cita).

"En las entidades del Estado las citas las daban con mucho tiempo de espera, y la incertidumbre creaba ansiedad..."

De acuerdo al impacto económico del diagnóstico del TEA en la familia, el 67\% indicó que es mayor el consumo, agrupándose en un $46 \%$ en la salud (terapia, medicamentos, estimulación temprana, entre otros), el 27\% alimentación, el 18\% transporte y $9 \%$ corresponde a actividades extracurriculares. Asimismo, un 67\% recibe ayuda de minusvalía con respeto a un 33\% que no la recibe.

En conclusión, los participantes hicieron énfasis en los aspectos que consideraron más importante en el proceso del diagnóstico del TEA, donde el 90\% es la comunicación acertada del diagnóstico precoz para establecer los procesos de atención temprana y ayudar a las familias a una mejor adaptación socioeconómica y psicológica.

"Es importante tener especialistas capacitados, para darle apoyo psicológico a la familia para superar el duelo del diagnóstico. Brindar asesoramiento y seguimiento de cómo sacar a la persona con Autismo adelante 
Invest. pens. crit. (ISSN 1812-3864)

Vol. 7, No. 1, enero- abril 2019

pp. 27-39

(estimulación temprana). Además, la sociedad debe estar informada o capacitada sobre qué es el Autismo., para que asi exista más tolerancia y empatía con los padres".

\section{Discusión}

El Trastorno del Espectro Autista (TEA) se caracteriza por una perturbación grave y generalizada en diversas áreas del desarrollo como son: las habilidades sociales, habilidades para la comunicación, intereses o conductas estereotipadas. Estas características por lo general se presentan durante los primeros años de vida, pero se tienden a confundir con una discapacidad intelectual, entre otras. (Martínez, A., \& Borges, A., 2012; Baña Castro, M., 2015). En la actualidad, el porcentaje de individuos afectados con el TEA en la población panameña es de $0.02 \%$ de la población total del país, sin embargo, el 66\% se sitúa entre los 6 y los 15 años de edad, y un 14.4\% entre 0 y 5 años de edad, es decir; que los niños con Autismo de este último grupo de edad están siendo atendidos en una proporción muy baja, o no se está diagnosticando a temprana edad. (Ministerio de Salud, 2013).

Con respecto al proceso del diagnóstico, el cuidador principal es la primera persona en darse cuenta de que algo está ocurriendo con el/la niño/a, (percibir conductas atípicas, como estereotipia, hiperactividad, auto-agresividad, escaso afecto, falta de atención, ausencia de contacto visual) (Dávalos Montalvo, 2015), estas sospechas se presentaron después del primer año de vida, no obstante en algunos casos el pediatra tardó un día o meses en la detección mientras otros "no se dieron cuenta del problema", trayendo como resultado un mayor número de visitas médicas en búsqueda de un diagnóstico certero y un aumento de múltiples calificaciones previas al Autismo como, por ejemplo: retraso mental, problemas de memoria, hiperactividad y dislexia, retrasando un diagnósticos certero y la estimulación temprana.

Al tener un diagnóstico claro del TEA, este tiene un impacto tanto en el individuo como en la familia, es decir, en el desenvolvimiento de las personas con TEA a nivel social, comunicativo, conductual y cognitivo (Taylor, Smiley, \& Richards, 2009), de igual manera a nivel familiar genera una huella en el ámbito socioeconómico, sociopsicológico, funcionamiento familiar y en el estado de salud. (Herrera, P., 2002), donde su interacción positiva o negativa establece que los individuos se repongan más rápido o más lento en tiempo.

En esta investigación se encontró que hubo un impacto Elevado, colocando de manifiesto a los individuos en el momento del diagnóstico, los cuales presentaron etapas de vulnerabilidad (Pozo, 2010), en las cuatro áreas (socioeconómico, sociopsicológico, funcionamiento familiar y en el estado de salud). Donde las áreas más sensibles fueron las funciones familiares (Comunicación familiar, afectividad, cohesión, armonía, roles) y la sociopsicológica (integración social, participación social, modo de vida familiar, convivencia social, planes y proyectos filiares). Esto quiere decir, que el impacto va depender en la forma de responder los familiares o acudientes ante el diagnóstico, debido a la poca información manejada, las creencias/mitos o la tardanza ante un diagnóstico certero para establecer pautas de intervención temprana.

Asimismo, se evidenció en los casos estudiados que, las estructuras familiares eran monoparentales, donde muchas veces el esfuerzo económico y sociopsicológico es mucho mayor, debido a la ausencia de otro tipo de ayudas. Por otro lado, los participantes, indicaron la importancia en la disminución del tiempo de espera en el servicio público para tener un diagnóstico precoz, y poder iniciar la atención 
Invest. pens. crit. (ISSN 1812-3864)

Vol. 7, No. 1, enero- abril 2019

pp. 27-39

temprana para el niño y ayudar a los familiares a una mejor adaptación familiar, sociopsicológica y socioeconómica (Sumalavia Casuso, M., 2019). Este proceso mejorará a medida que haya más especialistas en todo el país y brinden mayor información para la sociedad en general, para que se promueva no solo el conocimiento del Autismo, sino que exista mayor tolerancia y empatía para facilitar la inclusión social tanto del niño como de sus familiares.

\section{Conclusiones y recomendaciones}

Este estudio permite entender/comprender la vivencia ante el proceso del diagnóstico del Trastorno del Espectro Autista (TEA), con el fin de mejorar las políticas públicas, tomando en cuenta los factores culturales, creencias, contextos sociodemográficos, y acceso al sistema sanitario especializado a nivel nacional. Además, es importante, facilitar a la sociedad, talleres, información descriptiva, para mejorar los procesos de inclusión social tanto de los niños como el de sus familiares, brindándoles actividades extracurriculares que les permitan desarrollar sus habilidades. Por otra parte, durante el proceso del diagnóstico, una información adecuada acerca del Autismo, permite mejorar las tomas de decisiones que pueden provocar un impacto positivo en el núcleo familiar.

\section{Agradecimientos}

A la Universidad Católica Santa María La Antigua (USMA), al Centro Ann Sullivan de la Ciudad de Panamá, Dr. Luis Carlos Herrera, en el asesoramiento de los aspectos metodológicos de la tesis. Sistema Nacional de Investigación (SNI).

\section{Bibliografía}

Álvarez-López, E., Paul, M., Barragán, J., Calderón, I., Torres, E., Beltran, L...Morgado, C. (2014). Autismo: Mitos y realidades científicas. Rev. Med, UV, 37-41.

APA (2014). Manual Diagnóstico y Estadístico de los Trastornos Mentales DSM-5. American Psychiatric Publishing, Washington D.C.

Ávila Beltrán, Flavia, \& Soliz, Henry. (2006). Impacto psicosocial del autismo en la familia. Gaceta Médica Boliviana, 29(1), 21-27.

Baña Castro, M. (2015). El Rol de la Familia en la Calidad de Vida y la Autodeterminación de las Personas con Trastorno del Espectro del Autismo. Ciencias Psicológicas; 9 (2): 323 - 336

Baron-Cohen, S. (2008). Autism and Asperger Syndrome. Oxford, Inglaterra: Oxford University Press.

Bossa, C. \& Callias, M. (2000). Autismo: breve revisão de diferentes abordagens. Psicol. Reflex. Crit, 13(1), 167-177.

Canal, R., Garcia, P., Touriño, E., Santos, J., Martín, M., Ferrari, M.,...Posada, M. (2006) La detección Precoz del Autismo. Rev. Intervención Psicosocial, 15 (1), 29-47.

Dávalos Montalvo, M. B. (2015). Miedos, mitos y cambios que ocurren en los padres con hijos diagnosticados con Trastorno de Espectro Autista. Universidad de las Américas, Quito. Recuperado de http://dspace.udla.edu.ec/handle/33000/3448

Hernández, R., Fernández, C y Baptista, P. (2010). Metodología de la Investigación. 5ta edición. Mac Graw Hill. México. 
Invest. pens. crit. (ISSN 1812-3864)

Vol. 7, No. 1, enero- abril 2019

pp. 27-39

Herrera Santí, P., González Benítez, I. \& Soler Cárdenas, S. (2002) Metodología Para Evaluar el Impacto de los Acontecimientos Vitales de la Familia en la Salud Mental. Revista Cubana de Medicina General Integral, 18(2), 169-172.

Hviid A, Hansen JV, Frisch M. \& Melbye M. (2019). Measles, Mumps, Rubella Vaccination and Autism: A Nationwide Cohort Study. Ann Intern Med. [Epub ahead of print] doi: 10.7326/M18-2101

Instituto de Salud Carlos III. (2015). Cuestionario para Familias de Personas con Autismo. Recuperado de: http://iier.isciii.es/autismo/pdf/aut_cuest.pdf

Lasso, M. (2013). Panamá no cuenta con una estadística de la población autista. Panamá América. Recuperado de http://www.panamaamerica.com.pa/content/panamá-no-cuenta-con-unaestad $\%$ C3\%ADstica-de-la-población-autista

Levy, S.E., Mandell, D.S. \& Schultz, R.T. (2009). Autism. Lancet, 374, 1627-1638. doi:10.1016/S0140-6736(09)61376-3

López, S., Rivas, R.M. \& Taboada, E.M. (2009). Revisiones sobre el autismo. Revista Latinoamericana de Psicología, 41(3), 555-570

Martínez, A., \& Borges, A. (2012). El Autismo Como Trastorno Generalizado del Desarrollo. Alemania: Académica Española

Martínez Carazo, P.C. (2006). El método de estudio de caso Estrategia metodológica de la investigación científica. pensamiento \& gestión, 20. Universidad del Norte, 165-193.

Martínez, M., \& Cruz, M. (2008) Acercamiento a la Realidad de las Familias de Personas con Autismo. Rev. Intervención Psicosocial., 17(2).

Mebarak, M., Martínez, M. y Serna, A. (2009). Revisión bibliográfico analítica acerca de las diversas teorías y programas de intervención del autismo infantil. Psicología desde el Caribe. Universidad del Norte, 24: 120-146.

Mesa-Hernández, F. (2015). Consecuencias sociales del autismo para los padres y las madres. Trabajo final de grado, Facultad de Ciencias Políticas y Sociales y de la Comunicación, Universidad de la Laguna, España.

Ministerio de Salud (2013). Primera Encuesta Nacional Sobre el Autismo. República de Panamá. Recuperado de http://caspan.gob.pa/wp-content/uploads/2015/06/PRIMERAENCUESTA-NACIONAL-SOBRE-AUTISMO-2013.pdf.pdf

Montiel-Nava, C., Peña, J. (2008) Epidemiological Findings of Pervasive Developmental Disorders in a Venezuelan Study. Autism, 12(2), 191-202.

Organización Mundial de la Salud, OMS. (2016) Trastornos del Espectro Autista. Recuperado de http://www.who.int/mediacentre/factsheets/autism-spectrum-disorders/es/

Pozo, P., Sarriá, E., \& Brioso, A. (2014). Family quality of life and psychological well-being in parents of children with autism spectrum disorders: A double ABCX model. Journal of Intellectual Disability Research, 58(5), 442-458. http://doi.org/10.1111/jir.12042

Sumalavia Casuso, M. (2019). Proceso de adaptación de padres y madres de hijos diagnosticados dentro del Trastorno del Espectro Autista. Universidad Peruana de Ciencias Aplicadas (UPC), Lima, Perú. doi: https://doi.org/10.19083/tesis/625079

Stamboulian, D. (2015) Una Mirada profunda sobre el autismo ¿Por qué está en aumento? Infobae.http://www.infobae.com/2015/04/18/1722286-una-mirada-profunda-el-autismopor-que-esta-aumento/

Taylor, R., Smiley, L., \& Richards, B. (2009). Estudiantes Excepcionales. México D.F. Mc Graw Hill.

Unesco (2005). Declaraciòn Universal Sobre Bioètica y Derechos Humanos. Recuperado de: 
Invest. pens. crit. (ISSN 1812-3864)

Vol. 7, No. 1, enero- abril 2019

pp. 27-39

http://portal.unesco.org/es/ev.php-

URL ID=31058\&URL DO=DO TOPIC\&URL SECTION=201.html

Universidad Catòlica Santa Marìa la Antigua (2012). Normas de Investigaciòn. Buenas Pràcticas èticas en la Investigaciòn. Recuperado de: http://usma.ac.pa/wpcontent/uploads/2015/09/reglamento-de-investigacion.pdf

Valdés, A., Cisneros, R., M., Vales, J. \& Martínez, E. (2009). Creencias Erróneas de Padres de Niños con Autismo. Psicología Iberoamericana, 17(1), 15-23.

Verán C, N. (2011). Niveles de alexitimia en figuras parentales de niños con trastornos del espectro autista (Tesis de Licenciada). Pontificia Universidad Católica del Perú. Perú.

Yin, R. K. (1984/1989). Case Study Research: Design and Methods, Applied social research Methods Series, Newbury Park CA, Sage 